\title{
Elastic magnetic electron scattering from deformed nuclei
}

\author{
P. Sarriguren* and D. Merino \\ Instituto de Estructura de la Materia, IEM-CSIC, Serrano 123, E-28006 Madrid, Spain \\ O. Moreno and E. Moya de Guerra \\ Departamento de Estructura de la Materia, Física Térmica y Electrónica, and IPARCOS, Facultad de Ciencias Físicas, \\ Universidad Complutense de Madrid, Madrid E-28040, Spain \\ D. N. Kadrev, A. N. Antonov, and M. K. Gaidarov \\ Institute for Nuclear Research and Nuclear Energy, Bulgarian Academy of Sciences, Sofia 1784, Bulgaria
}

(Received 26 November 2018; published 22 March 2019)

\begin{abstract}
Magnetic form factors corresponding to elastic electron scattering from odd- $A$ nuclei are presented. The calculations are carried out in plane-wave Born approximation. The one-body properties are obtained in a deformed self-consistent mean-field calculation based on a Skyrme HF+BCS method. Collective effects are also included in the cranking approximation. Results on several stable nuclei are compared with the available experimental information. It is shown that a deformed formalism improves the agreement with experiment in deformed nuclei, while reproducing equally well spherical nuclei by taking properly the spherical limit of the deformed model and the effect of nucleon-nucleon correlations. Thus, the capability of the model to describe magnetic form factors is demonstrated. This opens the door to explore also unstable nuclei of particular interest that could be measured in future experiments on electron-radioactive beam colliders.
\end{abstract}

DOI: 10.1103/PhysRevC.99.034325

\section{INTRODUCTION}

Electron scattering is a unique tool for studying the electromagnetic properties of nuclei, getting insight into the nuclear charge and current distributions. There are several reasons for using electrons as probes. First, the electron interacts with the nucleus with the electromagnetic force, which is the best known interaction, accurately described by quantum electrodynamics. The coupling constant of the interaction is also sufficiently weak to not significantly disturb the nuclear structure under study. In addition, the weakness of the interaction allows one to work in first-order perturbation within the one-photon exchange approximation. Second, in contrast to the case of real photons, one can vary the energy transfer and the momentum transfer independently, thus mapping out the Fourier transform of the densities. Extensive work on electron scattering has been performed in the past in the elastic, inelastic, and quasielastic regimes, providing us with the most accurate measurements on charge radii, transition probabilities, as well as momentum distributions and spectroscopic factors [1-6].

Electron scattering is not only sensitive to the nuclear charge distribution. Electrons also scatter from the nuclear electromagnetic current distributions and the experimental observation of this process provides information on the convection and magnetization currents within the nucleus. In particular, elastic magnetic electron scattering provides fine

\footnotetext{
*p.sarriguren@csic.es
}

details of the nuclear ground-state convection and magnetization current distributions [7-9].

Although magnetic scattering shares the advantages of the electromagnetic probes, there are significant differences with respect to charge scattering. Because the angular momenta of the nucleons pair off within the core, the magnetic properties in odd- $A$ nuclei are determined to a large extent by the unpaired nucleon. Therefore, magnetic scattering mainly provides information on the single-particle properties of the valence nuclear wave function, whereas the collective aspects that dominate charge scattering show up only in particular cases. In addition, the intrinsic magnetic moments of protons and neutrons are quite similar in magnitude and thus, magnetic electron scattering will provide information on both, contrary to the case of charge scattering, which is mostly sensitive to protons.

Electron scattering experiments have been limited so far to stable isotopes, but the advantages of electron probes mentioned above can be exploited to gain information on unstable nuclei as well. At present, there is a large activity devoted to investigate with such probes the properties of stable [10] and unstable isotopes at radioactive nuclear beam facilities [11]. The technical challenges to perform such experiments on unstable nuclei are being considered in new facilities such as ELISe (FAIR-GSI) [12] and SCRIT (RIKEN) [13]. The conceptual design and the scientific challenges of the electronion collider ELISe (Electron-Ion Scattering in a Storage Ring) can be found in Ref. [14]. In SCRIT (Self-Confining Radioactive Ion Target), a circulating beam of electrons scatters off ions stored in a trap $[15,16]$, and the first elastic electron 
scattering experiment on ${ }^{132} \mathrm{Xe}$ has already been performed [17].

New interesting and challenging aspects such as nuclear halos and neutron skins can be addressed with these new electron scattering experiments from exotic nuclei. More generally, the evolution of the charge distributions in isotopic chains can give valuable information on the extent to which these phenomena may trigger sizable changes in the charge radius. This can be a test of the different theoretical models used for predicting charge distributions. Examples of these studies can be found in Refs. [18-27].

Similarly to the case of charge scattering, magnetic scattering has been also studied in stable nuclei from different theoretical frameworks [7], including shell model [28-31], relativistic mean-field [32-35], and deformed mean-field models [8,9,36-40].

Since the magnetic form factors are mainly determined by the last occupied orbital of the nucleon, valuable information on the characteristics of these single-particle properties have been extracted on stable nuclei. These experiments could become in the future an alternative tool to study the valence structure of odd- $A$ exotic nuclei, in particular, the wave function of the loosely bound halo nucleons that may be only slightly perturbed by the electromagnetic probe. Electron scattering would be an additional tool to complement the information obtained by other means. This is for example the case of magnetic dipole (M1) excitations in nuclei, where it is well known [41,42] that complementary information is obtained when using different electromagnetic $\left(\gamma, \gamma^{\prime}\right),\left(e, e^{\prime}\right)$ or hadronic $\left(p, p^{\prime}\right)$ probes.

Deformation can be an important issue when dealing with the structural evolution in isotopic chains, including both stable and unstable isotopes, undergoing a shape transition. A model describing properly the isotopic evolution of these properties is desirable.

The aim of this work is to analyze the dependence on deformation of the magnetic form factors of odd- $A$ nuclei and to show the capability of our deformed formalism to describe both spherical and deformed nuclei. To fulfill this purpose, we compare our results with the available experimental information on stable nuclei.

The paper is organized as follows. In the next section we present the theoretical formalism to calculate the magnetic form factors in deformed nuclei, as well as its spherical limit. Section III contains the results obtained in the case of spherical and deformed nuclei. Section IV contains the conclusions of the work.

\section{THEORETICAL FORMALISM}

\section{A. Cross section and form factors}

The formalism of electron scattering from deformed nuclei that we follow in this work was introduced and discussed earlier [8,36,37]. In particular, the work in Refs. [37,38] demonstrated the need for deformation to account for the data in ${ }^{181} \mathrm{Ta}$. Actually, magnetic scattering on ${ }^{181} \mathrm{Ta}$ was a case of success where first came theory and then experiment [43] confirmed the theoretical predictions in Ref. [37]. The method has been already applied to different cases $[39,40,44,45]$, where the various sensitivities of the results to different approximations concerning nuclear structure and reaction mechanism were studied. In particular, it was shown that the magnetic form factors of deformed nuclei may differ considerably from those of spherical nuclei. The sensitivity tests can be further explored, but the focus in this paper is mainly to investigate the dependence of the form factors on deformation.

Here, we briefly summarize this formalism. Following the notation of Ref. [8], the general cross section for electron scattering of ultrarelativistic electrons for transitions from the nuclear ground state $\left(I_{i}\right)$ to final states $\left(I_{f}\right)$ is given in the plane-wave Born approximation (PWBA) by

$$
\left.\frac{d \sigma}{d \Omega}\right|_{I_{i} \rightarrow I_{f}}=4 \pi \sigma_{M} f_{\mathrm{rec}}^{-1}\left[V_{L}\left|F_{L}\right|^{2}+V_{T}\left|F_{T}\right|^{2}\right],
$$

in terms of the Mott cross section

$$
\sigma_{M}=\left[\frac{\alpha \cos (\theta / 2)}{2 \epsilon_{i} \sin ^{2}(\theta / 2)}\right]^{2},
$$

and the recoil factor,

$$
f_{\mathrm{rec}}=1+\frac{2 \epsilon_{i} \sin ^{2}(\theta / 2)}{M_{\text {Target }}} .
$$

The dependence on the electron kinematics is given by the longitudinal and transverse Rosenbluth factors,

$$
V_{L}=\left(Q^{2} / q^{2}\right)^{2}, \quad V_{T}=\tan ^{2}(\theta / 2)-\left(Q^{2} / 2 q^{2}\right),
$$

where the kinematical variables are defined so that an incident electron with four-momentum $k_{i \mu}=\left(\epsilon_{i}, \mathbf{k}_{\mathbf{i}}\right)$ is scattered through an angle $\theta$ to four-momentum $k_{f \mu}=\left(\epsilon_{f}, \mathbf{k}_{\mathbf{f}}\right)$ by exchanging a virtual photon with four-momentum $Q=(\omega, \mathbf{q})$. The cross section can be separated into longitudinal (L) or Coulomb, and transverse (T) parts, weighted with different kinematical factors.

Because all the charged nucleons contribute equally to the Coulomb (charge) form factors $F_{L}$, they scale like $Z^{2}$ in the cross section. On the other hand, transverse form factors $F_{T}$ are basically single-particle observables that depend mostly on the properties of the unpaired nucleon in the outermost shell and do not carry this factor. As a consequence, $F_{L}$ dominates at most angles and special kinematic conditions are needed to maximize magnetic $F_{T}$ contributions. This is why backward scattering $\left(\theta=180^{\circ}\right)$ is commonly used to measure $F_{T}$ [see Eqs. (1)-(4)]. If magnetic form factors were to be measured in unstable nuclei, this difficulty of backward scattering will add to those inherent to the scattering from radioactive beams. In electron scattering experiments from unstable nuclei, the number of targets available is an important issue due to the limitations in the number of nuclei that can be produced and to their short half-lives. The luminosity $\mathcal{L}$, defined as the ratio of the event rate per time to the cross section, is a key parameter to asses the feasibility of the experiment. Luminosities over $10^{27} \mathrm{~cm}^{-2} \mathrm{~s}^{-1}$ have been already achieved at the SCRIT facility in the ${ }^{132}$ Xe experiment [17] and similar luminosities are expected for unstable nuclei in the next future [16] that will allow to measure them. The 
luminosities expected in ELISe are also within similar ranges [14].

The dependence on the nuclear structure is contained in the $q$-dependent longitudinal and transverse form factors, which are written in terms of Coulomb $(\mathrm{C})$, transverse electric $(\mathrm{E})$, and transverse magnetic (M) multipoles,

$$
\left|F_{L}\right|^{2}=\sum_{\lambda \geqslant 0}\left|F^{C \lambda}\right|^{2}, \quad\left|F_{T}\right|^{2}=\sum_{\lambda \geqslant 1}\left[\left|F^{M \lambda}\right|^{2}+\left|F^{E \lambda}\right|^{2}\right],
$$

which are defined as the reduced matrix elements of the multipole operators $\hat{T}^{\sigma \lambda}$ between initial and final nuclear states

$$
\left|F^{\sigma \lambda}\right|^{2}=\frac{\left|\left\langle I_{f} \| \hat{T}^{\sigma \lambda}(q)|| I_{i}\right\rangle\right|^{2}}{2 I_{i}+1} .
$$

For elastic scattering, parity and time reversal invariance imply that only the even Coulomb and odd transverse magnetic multipoles contribute. Then, at $\theta=180^{\circ}$ only odd magnetic multipoles will survive in PWBA,

$$
\left|F_{T}(q)\right|^{2}=\sum_{\lambda=\text { odd }}\left|F^{M \lambda}\right|^{2} .
$$

The magnetic multipole operators are defined as

$$
\hat{T}_{\mu}^{M \lambda}(q)=i^{\lambda} \int d \mathbf{r} j_{\lambda}(q r) \mathbf{Y}_{\lambda \lambda}^{\mu}\left(\Omega_{r}\right) \cdot \hat{\mathbf{J}}(\mathbf{r})
$$

where $\hat{\mathbf{J}}(\mathbf{r})$ is the current density operator. The currents $\hat{\mathbf{J}}$ in the transverse form factors contain both convection and magnetization components that arise from the motion and from the intrinsic magnetic moments of the nucleons, respectively.

When we calculate the total form factors, we include center of mass (c.m.) and finite nucleon size corrections. For the c.m. correction we use the usual factor obtained in the harmonicoscillator approximation,

$$
f_{\text {c.m. }}=\exp \left[b^{2} q^{2} /(4 A)\right],
$$

with the oscillator length $b=A^{1 / 6} \mathrm{fm}$.

In the magnetization currents, we use bare nucleon magnetic moments, $\mu_{s}^{p}=2.793 \mu_{N}, \mu_{s}^{n}=-1.913 \mu_{N}$, corrected with dipole form factors [46],

$$
G_{\tau}^{M}\left(q^{2}\right)=\mu_{s}^{\tau}\left[1+q^{2} /\left(18.23 \mathrm{fm}^{-2}\right)\right]^{-2} .
$$

In the convection currents, we use bare orbital $g$ factors, $g_{\ell}^{p}=1$ and $g_{\ell}^{n}=0$ scaled by $q$-dependent form factors. The proton electric form factor is given by a sum of monopoles parametrized in Ref. [46],

$$
G_{p}^{E}\left(q^{2}\right)=\sum_{n=1}^{4} \frac{a_{n}}{1+q^{2} / m_{n}^{2}} .
$$

The neutron electric form factor is given by the difference of two Gaussians [47],

$$
G_{n}^{E}\left(q^{2}\right)=\exp \left(-q^{2} r_{+}^{2} / 4\right)-\exp \left(-q^{2} r_{-}^{2} / 4\right)
$$

with $r_{\mp}^{2}=0.507413 \pm 0.038664 \mathrm{fm}^{2}$.

The effects of Coulomb distortion can be treated in a quantitative way in the distorted-wave Born approximation (DWBA) with a phase-shift calculation [48]. Nevertheless, for the analysis and interpretation of experimental data on magnetic scattering, neglecting the Coulomb distortion offers clear advantages and PWBA is commonly used. In PWBA the connection between data and the underlying physical quantities is more transparent, and calculations are much easier. Therefore, it is convenient to convert first the experimental magnetic cross sections into plane-wave form factors that can be used in PWBA interpretation. The most important effect of Coulomb distortion can be accounted for by using an effective momentum transfer. This procedure was done in the data used in this paper (taken from Ref. [7]) and therefore, the experimental form factors were converted into plane-wave form factors that can be directly compared with PWBA calculations.

It is worth noting that, in DWBA, backward electron scattering may receive also longitudinal contributions that could mix with the transverse ones. Then, a careful subtraction of longitudinal contributions should, in principle, be done before interpreting the backward scattering as purely transverse. These effects were studied in Ref. [49] concluding that on heavy deformed nuclei the distortion effects enhance somewhat the cross section at low $q$, while PWBA is reliable at larger $q$ values beyond $1 \mathrm{fm}^{-1}$. For the medium-mass nuclei and magnetic multipoles considered in this work, these effects are not expected to be important.

\section{B. Form factors in a deformed formalism}

The ground state of axially symmetric deformed nuclei is characterized by angular momentum $I$, projection along the symmetry axis $k$, and parity $\pi$. Initial and final states in Eq. (6) are the same for elastic scattering $\left(I k^{\pi}\right)$.

The magnetic $F^{M \lambda}$ multipole form factors can be written in terms of intrinsic form factors $\mathcal{F}^{M \lambda}$ weighted by angular momentum-dependent coefficients. To lowest order in an expansion in powers of the total angular momentum, the intrinsic multipoles depend only on the intrinsic structure of the ground-state band [8,9]. The transition multipoles in Eq. (7) for the elastic case $I_{f}=I_{i}=k$ can be written as

$$
\begin{aligned}
\left.F^{M \lambda}\right|_{\text {def }}= & \langle k k \lambda 0 \mid k k\rangle \mathcal{F}_{k}^{M \lambda}+\langle k-k \lambda 2 k \mid k k\rangle \mathcal{F}_{2 k}^{M \lambda} \\
& +\frac{\lambda(\lambda+1)}{\sqrt{2}}\langle k k \lambda 0 \mid k k\rangle \mathcal{F}_{R}^{M \lambda} .
\end{aligned}
$$

$\mathcal{F}_{R}^{M \lambda}$ are the transverse multipoles of the collective rotational current (rotational multipoles) that depend on the nuclear rotational model used to describe the band [8,9]. The singleparticle multipoles $\mathcal{F}_{k}^{M \lambda}$ and $\mathcal{F}_{2 k}^{M \lambda}$ depend only on the singleparticle intrinsic wave function of the odd nucleon if the even-even core is time-reversal invariant, as we assume in this work. They are different from zero only for $k \neq 0$ bands and are given by,

$$
\begin{aligned}
& \mathcal{F}_{k}^{M \lambda}=\left\langle\phi_{k}\left|\hat{T}_{0}^{M \lambda}\right| \phi_{k}\right\rangle, \\
& \mathcal{F}_{2 k}^{M \lambda}=\left\langle\phi_{k}\left|\hat{T}_{2 k}^{M \lambda}\right| \phi_{\bar{k}}\right\rangle+\delta_{k, 1 / 2} \frac{a}{\sqrt{2}} \mathcal{F}_{R}^{M \lambda} .
\end{aligned}
$$

$\hat{T}_{\mu}^{M \lambda}$ is the $\mu$ component of the $M \lambda$ tensor operator, see Eq. (8) and Ref. [2]. $\phi_{k}$ and $\phi_{\bar{k}}$ are the wave functions of the odd nucleon and its time reverse, respectively, and $a=\left\langle\phi_{k}\left|j_{+}\right| \phi_{\bar{k}}\right\rangle$ is the decoupling parameter for $k=1 / 2$ bands. 
As we can see from the above expressions, the magnetic form factors in odd- $A$ nuclei receive two types of contributions, single-particle and collective. While the former depends strongly on the single-particle state occupied by the unpaired nucleon, the latter depends on the rotational properties of the even-even core. The interplay between these two types of contributions and their relative intensities were discussed in Ref. [40]. It was found that the single-particle form factors are dominant at most $q$ values. They depend on the quantum number $k$ of the band, on the neutron or proton character of the odd nucleon, as well as on the mean field used to generate the single-particle states. The coupling of the unpaired nucleon to the deformed core and the currents connected with the collective rotation play also a role.

In the HF+BCS method for axially symmetric deformed nuclei, the wave function for the $i$ state is written in terms of the spin components $\phi_{i}^{+}$and $\phi_{i}^{-}$as [50],

$$
\begin{aligned}
\phi_{i}(\mathbf{R}, \sigma)= & \phi_{i}^{+}(r, z) \exp \left(\mathrm{i} \Lambda^{-} \varphi\right) \chi_{+}(\sigma) \\
& +\phi_{i}^{-}(r, z) \exp \left(\mathrm{i} \Lambda^{+} \varphi\right) \chi_{-}(\sigma) .
\end{aligned}
$$

The variables $r, z$, and $\varphi$ are the cylindrical coordinates of the radius vector $\mathbf{R} \cdot \chi_{ \pm}(\sigma)$ are the spin wave functions and $\Lambda^{ \pm}=$ $\Omega_{i} \pm 1 / 2 \geqslant 0$, where $\Omega_{i}$ is the projection along the symmetry axis of the total angular momentum, and it characterizes the single-particle Hartree-Fock solutions for axially symmetric deformed nuclei, together with parity $\pi_{i}$.

The wave functions $\phi_{i}$ are expanded into eigenfunctions $\psi_{\alpha}(\mathbf{R}, \sigma)$ of an axially deformed harmonic oscillator potential,

$$
\phi_{i}(\mathbf{R}, \sigma)=\sum_{\alpha} C_{\alpha}^{i} \psi_{\alpha}(\mathbf{R}, \sigma),
$$

with $\alpha=\left\{n_{r}, n_{z}, \Lambda, \Sigma\right\}$.

In the present work, the mean field of the odd- $A$ nucleus is generated within the equal filling approximation (EFA), a prescription used in self-consistent mean-field calculations that preserves time-reversal invariance. In this approximation half of the unpaired nucleon sits in a given orbital and the other half in the time-reversed partner. The odd nucleon orbital, characterized by $\Omega_{i}=k$ and $\pi_{i}$, is chosen among those around the Fermi level, according to the experimental spin and parity values. The EFA should be in our view the first reasonable attempt to describe odd- $A$ nuclei because of its numerical advantages and of its reliability when comparing with the results obtained from more sophisticated approaches. Such a comparison has been carried out in Ref. [51], where the EFA was compared with the exact blocking procedure with time-odd mean fields fully taken into account. It was shown that both procedures are strictly equivalent when time-odd terms are neglected. Different prescriptions for the time-odd coupling constants were also explored in Ref. [51], showing that the impact of the time-odd terms is quite small. The final conclusion was that the EFA is sufficiently precise for most practical applications. A microscopic justification of the EFA was given in Ref. [52], in terms of standard procedures of quantum statistical mechanics.

Time-odd fields vanish in the ground state of even-even nuclei, but they are different from zero in $I>0$ nuclei, where the time-reversal symmetry is broken. Our knowledge of the coupling constants of the time-odd terms is unfortunately still very limited. They could be constrained by experimental data on odd- $A$ nuclei, but their ability to do so is still an open issue [53,54]. It would be certainly interesting in the future to check the impact of going beyond the EFA by including explicitly odd terms in the Skyrme interactions to be adjusted to data on elastic magnetic electron scattering from odd- $A$ nuclei. All the results presented in this work correspond to the Skyrme interaction SLy4 [55], which has been thoroughly tested on many nuclear properties along the full nuclear chart.

The explicit expressions for all the intrinsic form factors in Eqs. (14) and (15) in terms of these wave functions can be found in Refs. $[8,37]$. Expressions for the intrinsic rotational multipoles $\mathcal{F}_{R}^{M \lambda}$ can be also found in Ref. [8,9] for different microscopic (projected Hartree-Fock and cranking), as well as macroscopic (rigid rotor and irrotational flow) models.

\section{Spherical limit}

In the spherical limit, the single-particle wave functions contain a single angular momentum component, so that the odd-nucleon wave function $\phi_{k}$ contains a single component $\phi_{j j}$. Thus, in this case $j=k=I_{i}$.

In this limit there are no collective magnetic multipoles $\left(\mathcal{F}_{R}^{M \lambda}=0\right)$ and the intrinsic single-particle form factors are given by the following expressions:

$$
\begin{aligned}
\mathcal{F}_{k}^{M \lambda} & =\left\langle\phi_{j j}\left|\hat{T}_{0}^{M \lambda}\right| \phi_{j j}\right\rangle \\
& =\frac{1}{\sqrt{2 j+1}}\langle j j \lambda 0 \mid j j\rangle\left\langle\phi_{j} \| \hat{T}^{M \lambda}|| \phi_{j}\right\rangle, \\
\mathcal{F}_{2 k}^{M \lambda} & =\left\langle\phi_{j j}\left|\hat{T}_{2 j}^{M \lambda}\right| \bar{\phi}_{j j}\right\rangle \\
& =\frac{(-1)^{\lambda}}{\sqrt{2 j+1}}\langle j-j \lambda 2 j \mid j j\rangle\left\langle\phi_{j}\left\|\hat{T}^{M \lambda}\right\| \phi_{j}\right\rangle .
\end{aligned}
$$

According to Eq. (6), for spherical nuclei one has

$$
\left.F^{M \lambda}\right|_{\mathrm{sph}}=\frac{1}{\sqrt{2 j+1}}\left\langle\phi_{j}\left\|\hat{T}^{M \lambda}\right\| \phi_{j}\right\rangle .
$$

On the other hand, using Eqs. (18) and (19), one can see that $\left.F^{M \lambda}\right|_{\text {def }}$ in Eq. (13) is related to $\left.F^{M \lambda}\right|_{\text {sph }}$ by a geometrical factor $\eta_{j}^{\lambda}$ given by

$$
\eta_{j}^{\lambda}=\langle j j \lambda 0 \mid j j\rangle^{2}\left[1+\delta_{\lambda, 2 j} \frac{\langle j-j \lambda 2 j \mid j j\rangle^{2}}{\langle j j \lambda 0 \mid j j\rangle^{2}}\right] .
$$

These coefficients are given explicitly by the following expressions for the two cases $\lambda<2 j$ and $\lambda=2 j$ :

$$
\begin{aligned}
\eta_{j}^{\lambda<2 j} & =\frac{(2 j+1) !(2 j) !}{(2 j+\lambda+1) !(2 j-\lambda) !}, \\
\eta_{j}^{\lambda=2 j} & =\frac{2 j+1}{4 j+1}\left[1+\frac{[(2 j) !]^{2}}{(4 j) !}\right] .
\end{aligned}
$$

In the next section, we denote by $\left.F^{M \lambda}\right|_{\text {sph limit }}$ the form factor obtained with the deformed codes for the case of odd- $A$ spherical nuclei using the above equations,

$$
\left.F^{M \lambda}\right|_{\text {sph limit }}=\left.\left[\eta_{j}^{\lambda}\right]^{-1} F^{M \lambda}\right|_{\text {def }},
$$


TABLE I. Calculated and measured charge root-mean-square radii $r_{c}$ [fm], quadrupole deformation parameter $\beta_{p}$, spectroscopic nuclear electric quadrupole moment $Q_{\text {lab }}[\mathrm{b}]$, and magnetic moments $\mu\left[\mu_{N}\right]$.

\begin{tabular}{lccccccccc}
\hline \hline Nucleus & $I^{\pi}$ & $r_{c, \text { th }}$ & $r_{c, \text { exp }}[63]$ & \multicolumn{1}{c}{$\beta_{p}$} & $Q_{\text {laboratory,th }}$ & $Q_{\text {laboratory,exp }}[64]$ & $\mu_{\text {th,sph }}$ & $\mu_{\text {th,def }}$ & $\mu_{\text {exp }}[64]$ \\
\hline${ }^{17} \mathrm{O}$ & $5 / 2^{+}$ & 2.7429 & $2.6932(75)$ & -0.034 & -0.009 & -0.02578 & -1.913 & -1.946 & $-1.89379(9)$ \\
${ }^{25} \mathrm{Mg}$ & $5 / 2^{+}$ & 3.0868 & $3.0284(22)$ & 0.338 & 0.167 & $+0.199(2)$ & -1.913 & -0.864 & $-0.85545(8)$ \\
${ }^{29} \mathrm{Si}$ & $1 / 2^{+}$ & 3.1434 & $3.1176(52)$ & -0.172 & - & - & -1.913 & -0.739 & $-0.55529(3)$ \\
${ }^{39} \mathrm{~K}$ & $3 / 2^{+}$ & 3.4509 & $3.4349(19)$ & 0.026 & 0.014 & $+0.0585(6)$ & +0.124 & +0.116 & $+0.39157(3)$ \\
${ }^{41} \mathrm{Ca}$ & $7 / 2^{-}$ & 3.4910 & $3.4780(19)$ & -0.020 & -0.027 & $-0.090(2) /-0.066(2)$ & -1.913 & -1.936 & $-1.5942(7)$ \\
${ }^{51} \mathrm{~V}$ & $7 / 2^{-}$ & 3.6233 & $3.6002(22)$ & -0.027 & -0.047 & $-0.043(5)$ & +5.793 & +5.817 & $+5.1487057(2)$ \\
${ }^{59} \mathrm{Co}$ & $7 / 2^{-}$ & 3.7891 & $3.7885(21)$ & 0.116 & 0.255 & $+0.41(1) /+0.35(3)$ & +5.793 & +5.152 & $+4.627(9)$ \\
${ }^{93} \mathrm{Nb}$ & $9 / 2^{+}$ & 4.3237 & $4.3240(17)$ & -0.042 & -0.216 & $-0.37(2) /-0.32(2)$ & +6.793 & +6.691 & $+6.1705(3)$ \\
${ }^{115} \mathrm{In}$ & $9 / 2^{+}$ & 4.6175 & $4.6156(26)$ & 0.090 & 0.632 & $+0.81(5)$ & +6.793 & +5.968 & $+5.5408(2)$ \\
\hline \hline
\end{tabular}

with $\left.F^{M \lambda}\right|_{\text {def }}$ as in Eq. (13) and $\eta_{j}^{\lambda}$ as in Eq. (21). These calculations are applied not only to the spherical nuclei $\left({ }^{17} \mathrm{O},{ }^{39} \mathrm{~K}\right.$, ${ }^{41} \mathrm{Ca}{ }^{51} \mathrm{~V}$, and $\left.{ }^{93} \mathrm{Nb}\right)$, but also to the deformed nuclei $\left({ }^{25} \mathrm{Mg}\right.$, ${ }^{29} \mathrm{Si},{ }^{59} \mathrm{Co}$, and ${ }^{115} \mathrm{In}$ ) in the limit of zero deformation, using constrained $\mathrm{HF}+\mathrm{BCS}$ solutions for $\beta=0$. The geometrical factors $\eta_{j}^{\lambda}$ in Eq. (24) are due to the loss of a favored intrinsic direction that takes place when one goes from the deformed to the spherical limit.

\section{Natural orbitals and nucleon correlation effects on electron elastic magnetic scattering from nuclei}

It is known (see, e.g., Refs. [56,57]) that many experimental data of scattering and reactions on nuclei show the existence of sizable nucleon-nucleon (NN) correlation effects on nuclear properties that cannot be described correctly within the mean-field approximation in the nuclear theory. This concerns mainly the form of the single-particle wave functions, the occupation probabilities, the spectral functions, the momentum distributions of nucleons and clusters, and others. It became possible to restore the single-particle picture in the methods in which the NN correlations are accounted for by using the so-called natural orbitals (NOs) and natural occupation probabilities within the NO representation [58] of the one-body density matrix (OBDM) that corresponds to the correlated ground state of the system. The NOs and the natural occupation numbers for the nucleus with $A$ particles can be obtained by the diagonalization of the OBDM in a given model solving the equation:

$$
\int d \mathbf{r}^{\prime} \rho\left(\mathbf{r}, \mathbf{r}^{\prime}\right) \psi_{\alpha}\left(\mathbf{r}^{\prime}\right)=n_{\alpha} \psi_{\alpha}(\mathbf{r})
$$

where $\psi_{\alpha}(\mathbf{r})$ are the NOs and $n_{\alpha}$ are the natural occupation numbers that fulfill the conditions:

$$
0 \leqslant n_{\alpha} \leqslant 1 ; \quad \sum_{\alpha} n_{\alpha}=A
$$

Thus, in the NO representation [58] the OBDM has the form:

$$
\rho\left(\mathbf{r}, \mathbf{r}^{\prime}\right)=\sum_{\alpha} n_{\alpha} \psi_{\alpha}^{*}\left(\mathbf{r}^{\prime}\right) \psi_{\alpha}(\mathbf{r})
$$

In the present work, the NOs related to the OBDM in the coherent density fluctuation model (CDFM) [56,57,59-61] are obtained for different nuclei and used in the calculations of the magnetic form factors.

The CDFM is based [56,59] on the $\delta$-function limit of the generator-coordinate method [62]. The OBDM in the model has the form:

$$
\rho\left(\mathbf{r}, \mathbf{r}^{\prime}\right)=\int|\mathcal{F}(x)|^{2} \rho_{x}\left(\mathbf{r}, \mathbf{r}^{\prime}\right) d x
$$

being an infinite superposition of the OBDMs $\rho_{x}\left(\mathbf{r}, \mathbf{r}^{\prime}\right)$ related to the one in the plane-wave case:

$$
\rho_{x}\left(\mathbf{r}, \mathbf{r}^{\prime}\right)=3 \rho_{0}(x) \frac{j_{1}\left(k_{F}(x)\left|\mathbf{r}-\mathbf{r}^{\prime}\right|\right)}{k_{F}(x)\left|\mathbf{r}-\mathbf{r}^{\prime}\right|} \Theta\left(x-\frac{\left|\mathbf{r}+\mathbf{r}^{\prime}\right|}{2}\right),
$$

with

$$
\begin{aligned}
& \rho_{0}(x)=\frac{3 A}{4 \pi x^{3}}, \\
& k_{F}(x)=\left(\frac{3 \pi^{2}}{2} \rho_{0}(x)\right)^{1 / 3} \equiv \frac{\alpha}{x}, \quad \alpha=\left(\frac{9 \pi A}{8}\right)^{1 / 3} .
\end{aligned}
$$

The weight function $|\mathcal{F}(x)|^{2}$ in (28) in the case of monotonically decreasing density distributions $(d \rho(r) / d r \leqslant 0)$ can be obtained from the density:

$$
|\mathcal{F}(x)|^{2}=-\left.\frac{1}{\rho_{0}(x)} \frac{d \rho(r)}{d r}\right|_{r=x} .
$$

The applications of the NOs to studies of the magnetic form factors are a part of general studies of the correlated OBDMs and their usage to analyses of nuclear states and processes.

\section{RESULTS AND DISCUSSION}

The nuclei studied here have been chosen because experimental information on elastic magnetic electron scattering form factors is available and a comparison with the calculated ones can be performed to test the reliability of the theoretical models.

Table I shows ground-state properties of the nuclei under study, namely, charge root-mean-square radii $r_{c}$, spectroscopic electric quadrupole moments $Q_{\text {lab }}$, and magnetic moments $\mu$. The results are compared with experimental data from Ref. [63] for radii and from [64] for quadrupole and magnetic moments. 
The relationship between the intrinsic quadrupole moment $Q_{0}$ and the quadrupole deformation parameter $\beta_{p}$ is given by

$$
Q_{0}=\sqrt{\frac{5}{\pi}} Z e\left\langle r^{2}\right\rangle \beta_{p}
$$

where $\left\langle r^{2}\right\rangle$ is the nuclear mean-square radius. The measured quadrupole moment $Q_{\text {lab }}$ is related to the intrinsic quadrupole moment $Q_{0}$ by

$$
Q_{\mathrm{lab}}=\frac{3 k^{2}-I(I+1)}{(I+1)(2 I+3)} e Q_{0} .
$$

The measured electric quadrupole moments in Table I correspond to ground states $I=k$. Of course, $Q_{\mathrm{lab}}=0$ for $I=k=$ $1 / 2$.

The magnetic moments in the deformed case $\mu_{\mathrm{th} \text {,def }}$ are obtained from the expression,

$\mu_{I}=g_{R} I+\frac{k^{2}}{I+1}\left[g_{k}-g_{R}+\delta_{k, 1 / 2}(2 I+1)(-1)^{I+1 / 2} \sqrt{2} g_{2 k}\right]$,

where $g_{R}, g_{k}$, and $g_{2 k}$ are defined in Ref. [8] and calculated in the cranking approximation. We also show for comparison the Schmidt values $\mu_{\text {th,sph }}$ obtained in the spherical limit.

The agreement between the calculated and the measured $r_{c}$ is quite good, especially in the medium-mass nuclei from ${ }^{29} \mathrm{Si}$ to ${ }^{115} \mathrm{In}$, where the error is less than $1 \%$. This error is slightly larger in the lighter nuclei. The quadrupole deformation parameters $\beta_{p}$ in Table I show that the isotopes ${ }^{17} \mathrm{O},{ }^{39} \mathrm{~K},{ }^{41} \mathrm{Ca}$, ${ }^{51} \mathrm{~V}$, and ${ }^{93} \mathrm{Nb}$ could be treated as spherical, while ${ }^{25} \mathrm{Mg}$, ${ }^{29} \mathrm{Si}$, ${ }^{59} \mathrm{Co}$, and ${ }^{115} \mathrm{In}$, should be treated as deformed. The calculated and measured $Q_{\text {lab }}$ agree in sign, as well as in magnitude in most cases.

Concerning magnetic moments, for the spherical nuclei $\left({ }^{17} \mathrm{O},{ }^{39} \mathrm{~K},{ }^{41} \mathrm{Ca},{ }^{51} \mathrm{~V}\right.$, and $\left.{ }^{93} \mathrm{Nb}\right)$, both the Schmidt and the deformed values show a reasonable agreement with the experimental data, except in the case of ${ }^{39} \mathrm{~K}$, where the calculations overestimate the data in both cases. On the other hand, for the deformed nuclei $\left({ }^{25} \mathrm{Mg},{ }^{29} \mathrm{Si},{ }^{59} \mathrm{Co}\right.$, and $\left.{ }^{115} \mathrm{In}\right)$, there is a clear improvement of the agreement with experiment in the deformed formalism.

The reason why deformation does not improve the results on spherical nuclei is simply that this is not a proper model to describe them. For odd- $A$ nuclei, the deformed model we use is valid for well-deformed nuclei where the coupling between the unpaired particle and the rotation of the even-even core can be treated in the rotor plus quasiparticle approximation (using Skyrme HF+BCS single-particle states). The spherical model for odd- $A$ nuclei assumes that the ground-state spin $I$ and parity $\pi$ are those of the single nucleon determined from the quantum numbers of the last filled spherical orbital, $I=j$, $\pi=(-1)^{\ell}$. Typically, the deformed model can be applied when the quadrupole deformation parameter $\beta$ is larger than 0.1 , whereas the spherical model is valid for zero or very small deformations. Nuclei with small intrinsic deformations, such as those in Table I $\left({ }^{17} \mathrm{O}, \beta=-0.034 ;{ }^{39} \mathrm{~K}, \beta=0.026 ;{ }^{41} \mathrm{Ca}\right.$, $\beta=-0.020 ;{ }^{51} \mathrm{~V}, \beta=-0.027 ;{ }^{93} \mathrm{Nb}, \beta=-0.042$ ) can be considered to be spherical or rather soft nuclei, where the

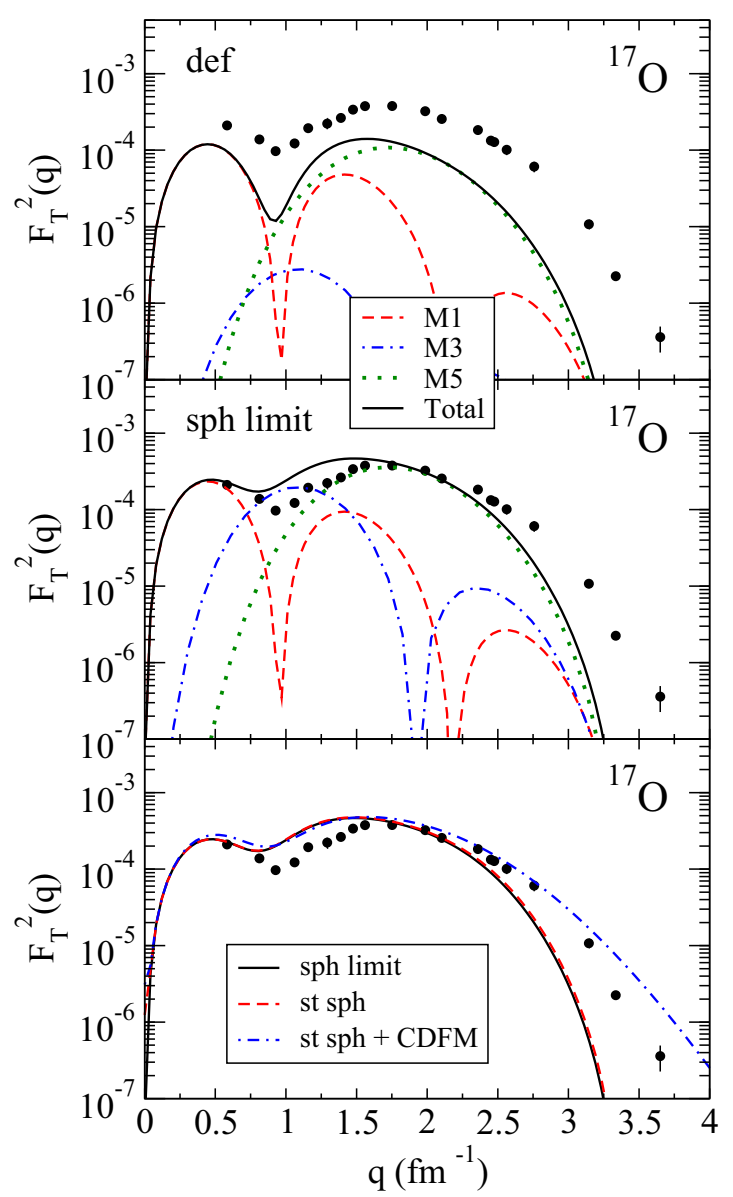

FIG. 1. Magnetic form factors of ${ }^{17} \mathrm{O}\left(I^{\pi}=5 / 2^{+}\right)$decomposed into M1, M3, and M5 multipole components in the deformed model (top) and in its spherical limit (middle). We also show (bottom) the results from standard spherical calculations alone and together with CDFM (see text). Data are taken from $[7,66]$.

deformed formalism does not imply necessarily an improvement over the spherical formalism. It is worth mentioning that in the case of ${ }^{93} \mathrm{Nb}$, where the deformed formalism improves somewhat the magnetic moment, the deformation is larger than in the other nuclei considered as spherical. The description of these soft nuclei could be refined with a more sophisticated treatment of the coupling of single-particle and collective aspects, but this is beyond the purpose of the present study. In any case, in the soft nuclei, the corrections introduced by deformation are rather small and do not change significantly the spherical results.

\section{A. Spherical nuclei}

In this section we study the magnetic form factors in several nuclei that according to Table I can be taken as spherical, namely, ${ }^{17} \mathrm{O},{ }^{39} \mathrm{~K},{ }^{41} \mathrm{Ca},{ }^{51} \mathrm{~V}$, and ${ }^{93} \mathrm{Nb}$.

In Figs. 1-5 we show the calculations from the deformed model in the top panels. The middle panels contain the results in the spherical limit defined by Eq. (24). The geometrical factors $\eta_{j}^{\lambda}$ relating the spherical and the deformed form factors in Eq. (21) are given explicitly in Table II for each $j$ state and 
TABLE II. Geometrical factors $\left[\eta_{j}^{\lambda}\right.$ in Eq. (21)] for $j$ states and $\lambda$ multipoles.

\begin{tabular}{|c|c|c|c|c|c|c|c|c|c|c|c|c|c|c|c|}
\hline$\lambda$ & 1 & 1 & 3 & 1 & 3 & 5 & 1 & 3 & 5 & 7 & 1 & 3 & 5 & 7 & 9 \\
\hline$\eta_{j}^{\lambda}$ & 1 & 0.60 & 0.60 & 0.7143 & 0.1190 & 0.5476 & 0.7778 & 0.2121 & 0.0163 & 0.5335 & 0.8182 & 0.2937 & 0.0419 & 0.0019 & 0.5263 \\
\hline
\end{tabular}

$\lambda$ multipole. It is worth noting that, in the spherical limit, there are no collective contributions $\mathcal{F}_{R}^{M \lambda}$ from rotations of the core. The bottom panels show a comparison between the results in the spherical limit (sph limit) with the results obtained from a standard spherical calculation (st sph) based on the code by Blok and Heisenberg [65]. This comparison helps us to check the reliability of the spherical limit. We also test the role of NN correlations (st sph $+\mathrm{CDFM}$ ) by incorporating these effects and comparing the results. Correlation methods beyond the mean-field picture are needed, especially to account for the behavior at high momentum transfer. The NO representation is a convenient way to deal with these correlations. In Ref. [57] NOs, obtained within the CDFM, were used to calculate electron magnetic scattering form factors in ${ }^{17} \mathrm{O}$ and ${ }^{41} \mathrm{Ca}$, which are examples of nuclei with a single nucleon

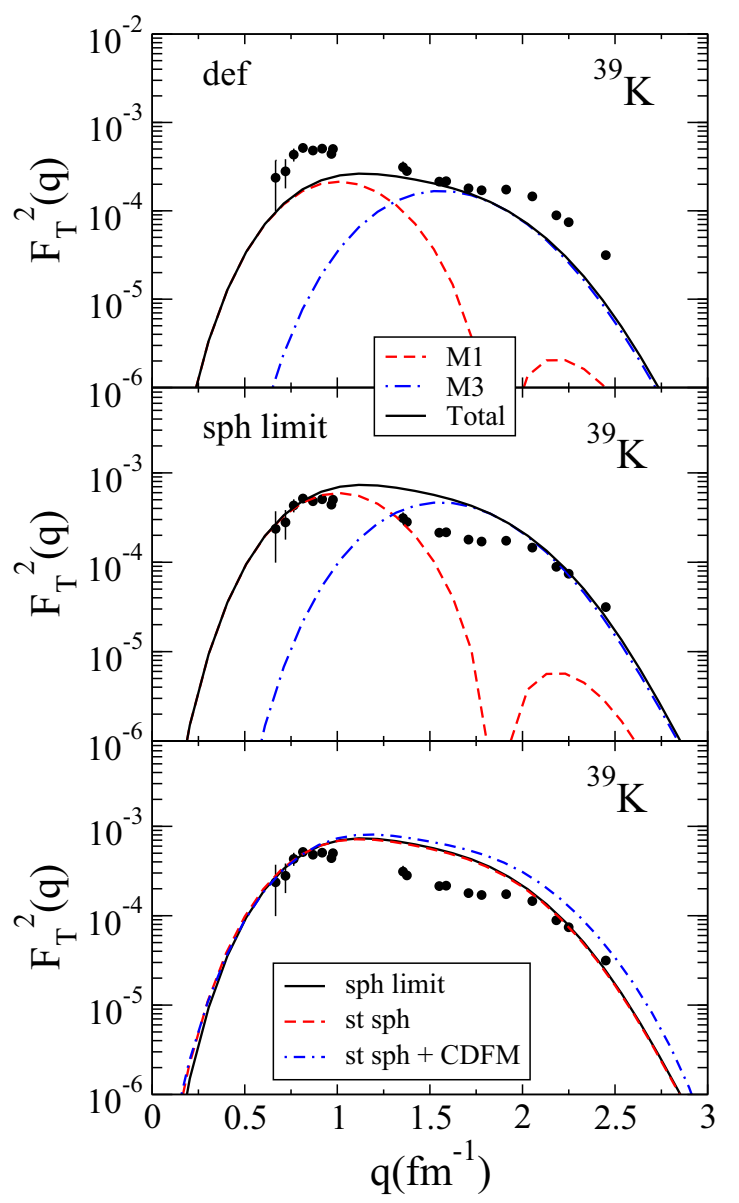

FIG. 2. Same as in Fig. 1, but for ${ }^{39} \mathrm{~K}\left(I^{\pi}=3 / 2^{+}\right)$decomposed into M1 and M3 multipole components. Data are taken from [7] and references therein. outside a doubly closed core. In this work we extend those calculations to other spherical nuclei.

Figure 1 for ${ }^{17} \mathrm{O}\left(I^{\pi}=5 / 2^{+}\right)$shows the first example of this comparison. Data are from Refs. [7,66]. In the deformed case, contributions from the M3 multipole are negligible due to the reduction factor $\eta_{j}^{\lambda}=0.119$, but in the spherical case it makes a difference by filling the region between the two peaks. The first peak is totally due to the M1 multipole, while the second peak and the tail at large $q$ is determined by the M5 multipole. The spherical description clearly improves the agreement with experiment due to the geometrical factors that scale the various multipoles. The agreement between the results from the spherical limit and the results from Ref. [65] is remarkable, which is the general trend observed in all cases studied in this subsection. The main effect of the NN

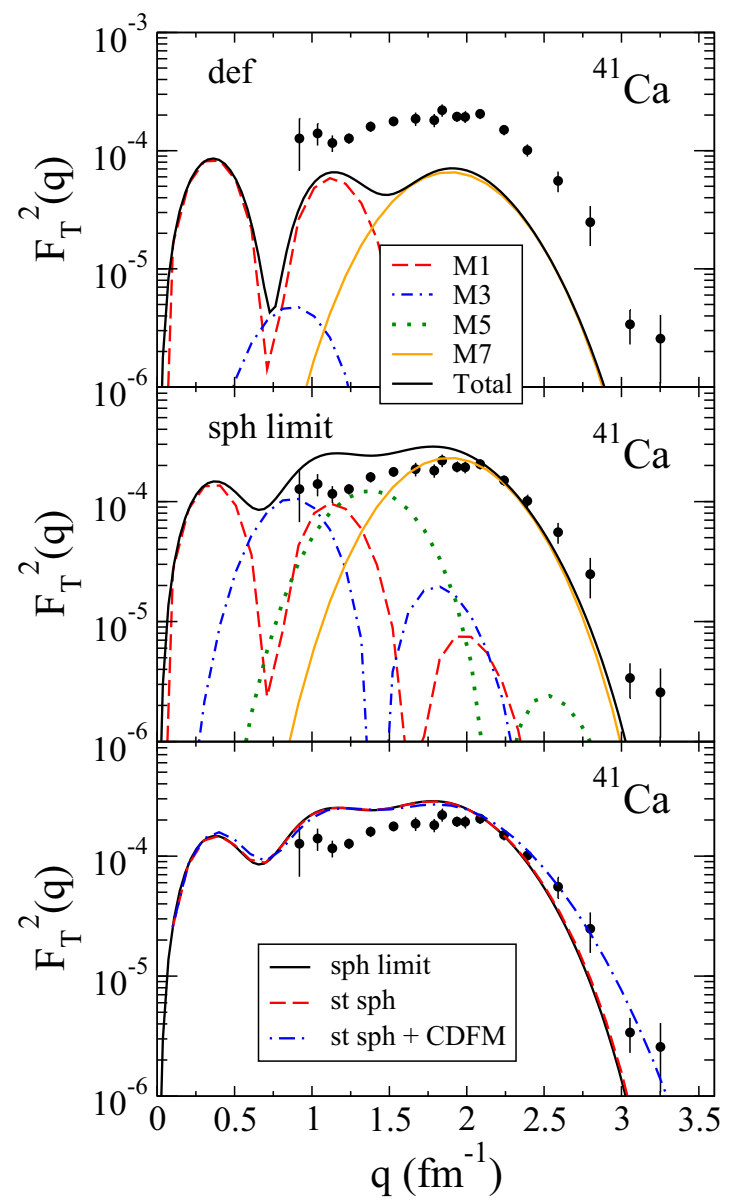

FIG. 3. Same as in Fig. 1, but for ${ }^{41} \mathrm{Ca}\left(I^{\pi}=7 / 2^{-}\right)$decomposed into M1, M3, M5, and M7 multipole components. Data are taken from Ref. [67]. 


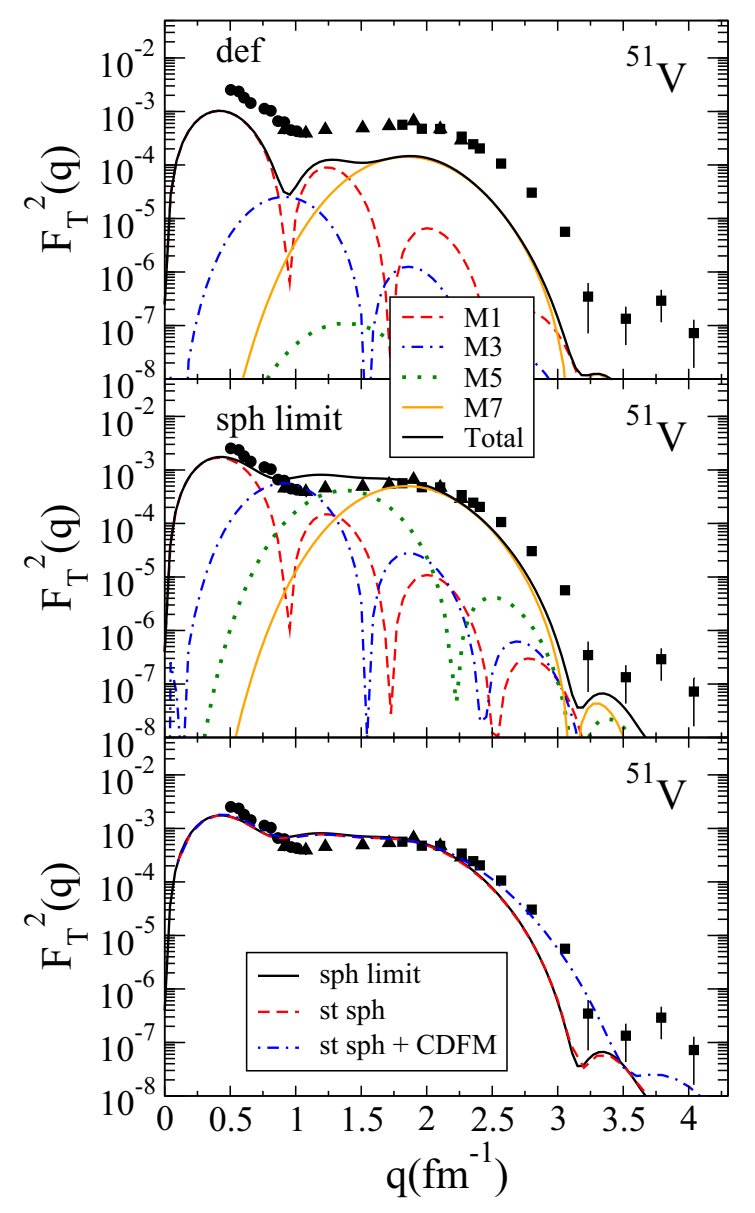

FIG. 4. Same as in Fig. 1, but for ${ }^{51} \mathrm{~V}\left(I^{\pi}=7 / 2^{-}\right)$decomposed into M1, M3, M5, and M7 multipole components. Data are taken from Refs. [7] (circles), [68] (triangles), and [69] (squares).

correlations calculated with natural orbits within the CDFM [57] is to modify the tails by shifting the form factors to higher $q$ values, thus improving the agreement with experiment. This feature is also observed in all cases studied.

Figure 2 shows the same results, but for ${ }^{39} \mathrm{~K}\left(I^{\pi}=3 / 2^{+}\right)$. Now, M1 (M3) determines the first (second) peak. The enhancement of the multipoles in the spherical limit improves somewhat the agreement with experimental data. The effect of the NN correlations is similar to the previous case. There is a difficulty in the description of the region $1<q<2 \mathrm{fm}^{-1}$, where the data are overestimated by the calculations. This is the region where the M3 multipole is dominant.

In Fig. 3 we show the results for ${ }^{41} \mathrm{Ca}\left(I^{\pi}=7 / 2^{-}\right)$. In the deformed case we get a three-peaked profile. The two first peaks are due to the M1 multipole, while the third peak is determined by M7. M3, and M5 represent a negligible contribution, partly due to their geometrical factors (see Table II). In the spherical limit all the multipoles, but especially M3 and M5, are enhanced with respect to the deformed case, producing a much better agreement with experiment. In particular, M3 and M5 fill the total form factor in the range between $0.5<q<2 \mathrm{fm}^{-1}$, getting the results closer to the data from Ref. [67], although slightly overestimating them

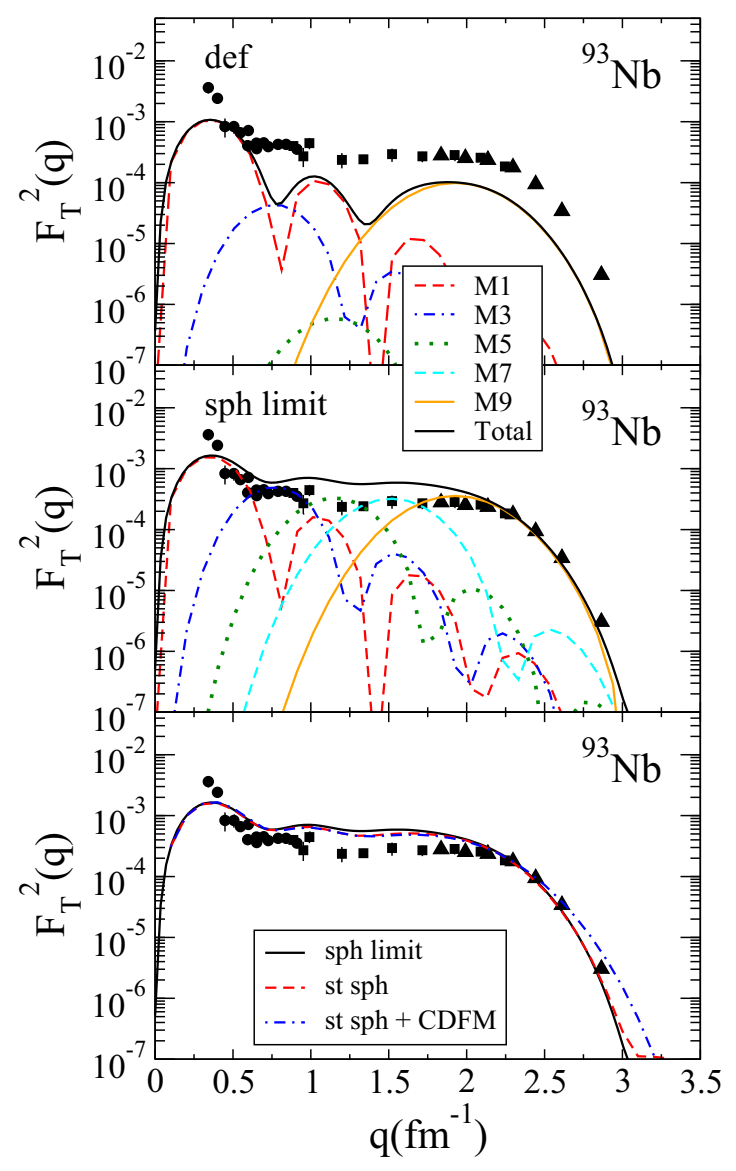

FIG. 5. Same as in Fig. 1, but for ${ }^{93} \mathrm{Nb}\left(I^{\pi}=9 / 2^{+}\right)$decomposed into M1, M3, M5, M7, and M9 multipole components. Data are taken from [7] (circles), [70] (squares), and [71] (triangles).

in the region $1<q<2 \mathrm{fm}^{-1}$. The tails are again improved when $\mathrm{NN}$ correlations are included.

Figure 4 shows the results for ${ }^{51} \mathrm{~V}\left(I^{\pi}=7 / 2^{-}\right)$with data from $[7,68,69]$. Again, M1 determines the first peak and M7 the broad second peak and the tail. The spherical limit with the multipoles enhanced reproduces better the data. The NN correlations improve the decay of the tail at large momentum transfer.

Finally, Fig. 5 for ${ }^{93} \mathrm{Nb}\left(I^{\pi}=9 / 2^{+}\right)$shows that, in the deformed case, M1 is responsible for the two first peaks in the form factor and M9 determines the third peak and the structure of the tail at high momentum transfer. The role of M3, M5, and M7 is irrelevant due to the reduction factors. In the spherical limit, all the multipoles play a role. M1 determines the region below $q=0.5 \mathrm{fm}^{-1}$, M3 between $q=0.5$ and $q=1 \mathrm{fm}^{-1}$, M5 between $q=1.0$ and $q=1.5 \mathrm{fm}^{-1}$, M7 between $q=$ 1.5 and $q=2.0 \mathrm{fm}^{-1}$, and finally M9 the behavior beyond $q=2.0 \mathrm{fm}^{-1}$. The final result is a smeared profile in much better agreement with the experiment $[7,70,71]$. The NN correlations improve slightly the agreement with experiment.

In summary, we have found that in the case of the odd- $A$ spherical nuclei studied, the spherical formalism reproduces quite reasonably the main features of the elastic magnetic form factors measured. The results from standard spherical 


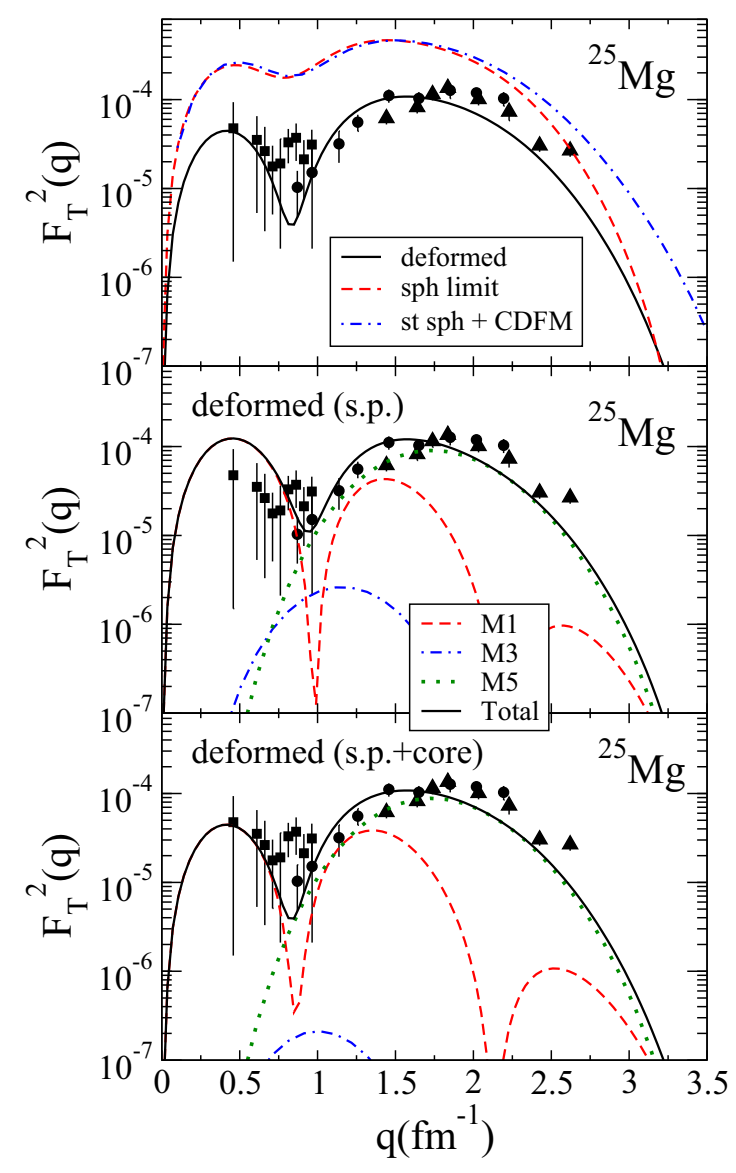

FIG. 6. Magnetic form factors of ${ }^{25} \mathrm{Mg}\left(I^{\pi}=5 / 2^{+}\right)$in the deformed formalism and in its spherical limit, as well as in the spherical CDFM (top). The multipole decomposition is shown in the deformed model for single-particle contributions (middle), and including collective contributions from the rotating core in the cranking approximation (bottom). Data used in Ref. [7] are taken from [70] (circles) and [72] at different kinematical conditions (squares and triangles).

formalism perfectly agree with the results obtained in the spherical limit of the deformed formalism. The latter is shown to be inadequate to describe spherical or soft nuclei with very small deformations, unless we previously rewrite the corresponding equations in the spherical limit. NN correlations modify the high- $q$ tails of the form factors ( $q$ beyond $2 \mathrm{fm}^{-1}$ ), improving the agreement with experiment. On the other hand, as we shall see in the next section, the deformed formalism is needed to describe properly the measured form factors, as it was also necessary to account for the values of the experimental magnetic moments in deformed nuclei.

\section{B. Deformed nuclei}

In the next figures, Figs. 6-9, we compare in the top panels calculations from the deformed and spherical formalisms in the case of deformed nuclei, that according to Table I are ${ }^{25} \mathrm{Mg},{ }^{29} \mathrm{Si},{ }^{59} \mathrm{Co}$, and ${ }^{115} \mathrm{In}$. In this case, to calculate the spherical limit a HF+BCS calculation constrained to zero deformation has been performed for each nucleus. In the middle panels we show the total magnetic form factor from

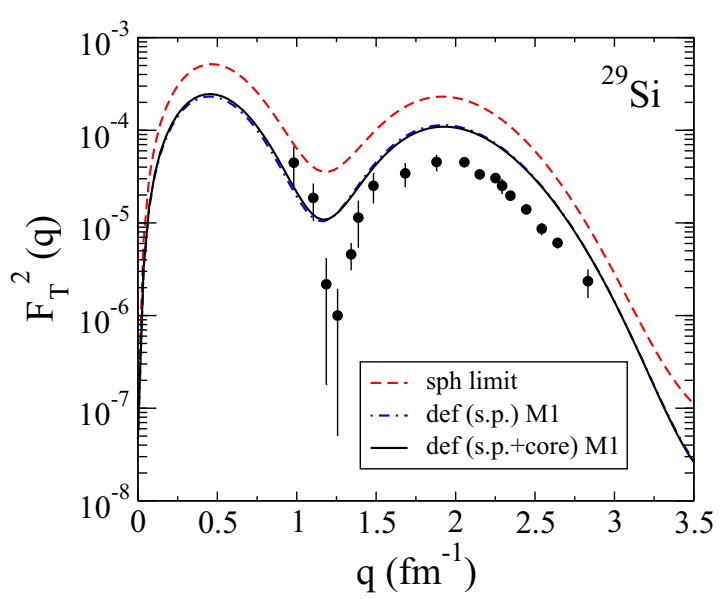

FIG. 7. Magnetic form factors of ${ }^{29} \mathrm{Si}\left(I^{\pi}=1 / 2^{+}\right)$in the deformed and spherical models. Only the M1 multipole contributes. Data are taken from Ref. [7] and references therein.

the deformed model decomposed into multipolarities with single-particle contributions only. The bottom panels show the results including rotational collective contributions from the core $\mathcal{F}_{R}^{M \lambda}$, calculated in the cranking model. Explicit expressions for these contributions can be seen in Ref. [8]. We studied in the past $[40,44]$ the effect of these collective rotational contributions from different microscopic and macroscopic models and concluded that they are, in general, small compared to single-particle contributions. They are only expected to show up in the M1 multipoles at low $q$ and do not differ much from one rotational model or another. Thus, we opted here for calculations from the cranking model that produce better moments of inertia.

Figure 6 shows the results for ${ }^{25} \mathrm{Mg}\left(I^{\pi}=5 / 2^{+}\right)$with data taken from Refs. [7,70,72]. In the top panel we show the deformed calculations compared with the spherical limit, as well as with the standard spherical calculation with CDFM correlations. We observe in the deformed case a clear improvement of the agreement with experiment. The first peak is due to M1, whereas the second is due to M5, with a negligible contribution from M3. We can see that the contribution of the core rotational currents appears mainly in the first peak, due to the effect on M1 at low $q$. This contribution improves the agreement in the first peak. The high- $q$ behavior is determined by the M5 multipole, which is practically unaffected by $\mathcal{F}_{R}^{M 5}$.

In the next figure, Fig. 7, we show the results for ${ }^{29} \mathrm{Si}$ $\left(I^{\pi}=1 / 2^{+}\right)$. In this case only the M1 multipole contributes and one panel is enough to show the various contributions. We get a much better agreement with data [7] in the deformed case. We should note that the improvement is due directly to the structure of the deformed orbital in comparison to the spherical, because in this case the M1 multipole is not reduced by the geometrical factor $\eta_{j}^{\lambda}$. There is practically no contribution from the core rotational current, even though in this case we have an extra contribution from the core proportional to the decoupling parameter $a=1.166$ [see Eq. (15)].

Figure 8 displays the results for the nucleus ${ }^{59} \mathrm{Co}\left(I^{\pi}=\right.$ $\left.7 / 2^{-}\right)$. The data from Refs. $[7,71,73]$ are better reproduced by 


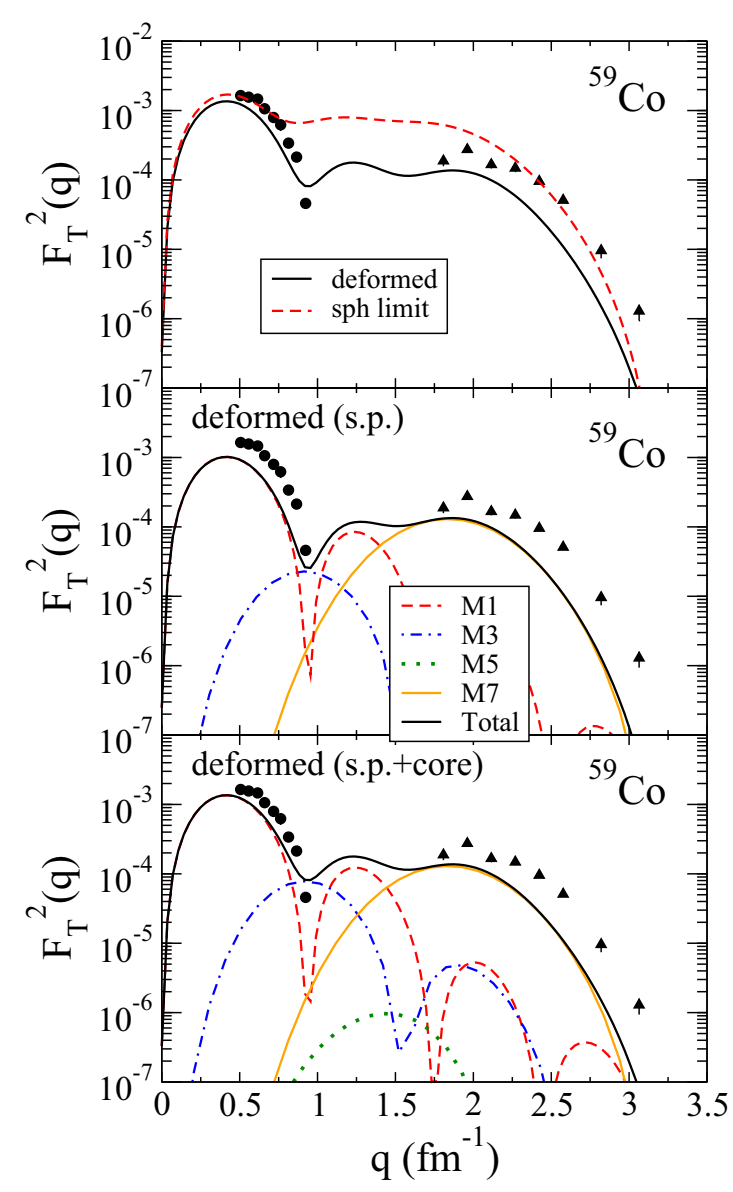

FIG. 8. Magnetic form factors of ${ }^{59} \mathrm{Co}\left(I^{\pi}=7 / 2^{-}\right)$in the deformed and spherical models (top). The multipole decomposition is shown in the deformed model for single-particle contributions (middle) and including collective contributions from the rotating core in the cranking approximation (bottom). Data used in Ref. [7] are taken from [73] (circles) and [71] (triangles).

the spherical case at low and high $q$ values, but the deformed calculations reproduce better the whole structure of the first peak including the fall of the curve. The filling of the form factors in the range $1<q<2 \mathrm{fm}^{-1}$ produced by the spherical calculation is not observed experimentally, which seems to favor the deformed picture. This could be an indication that we are dealing in this case with small deformations, see Table I. We can see again a small contribution from the core rotational current in the first peak that improves slightly the agreement with experiment.

In the case of ${ }^{115}$ In $\left(I^{\pi}=9 / 2^{+}\right)$, Fig. 9, the data from Ref. [7] seem to show three peaks, which are better described in the deformed picture. The spherical description smears too much the profile of the curve. In this nucleus, collective effects do not play a significant role.

It is worth noting that in the cases of ${ }^{59} \mathrm{Co}$ and ${ }^{115} \mathrm{In}$, the form factors at low $q$ that determine the magnetic moment of the nucleus are quite similar in the cases of spherical and deformed calculations, with somewhat larger values in the spherical case. This is correlated to the values of the magnetic moments quoted in Table I. We can see that the magnetic

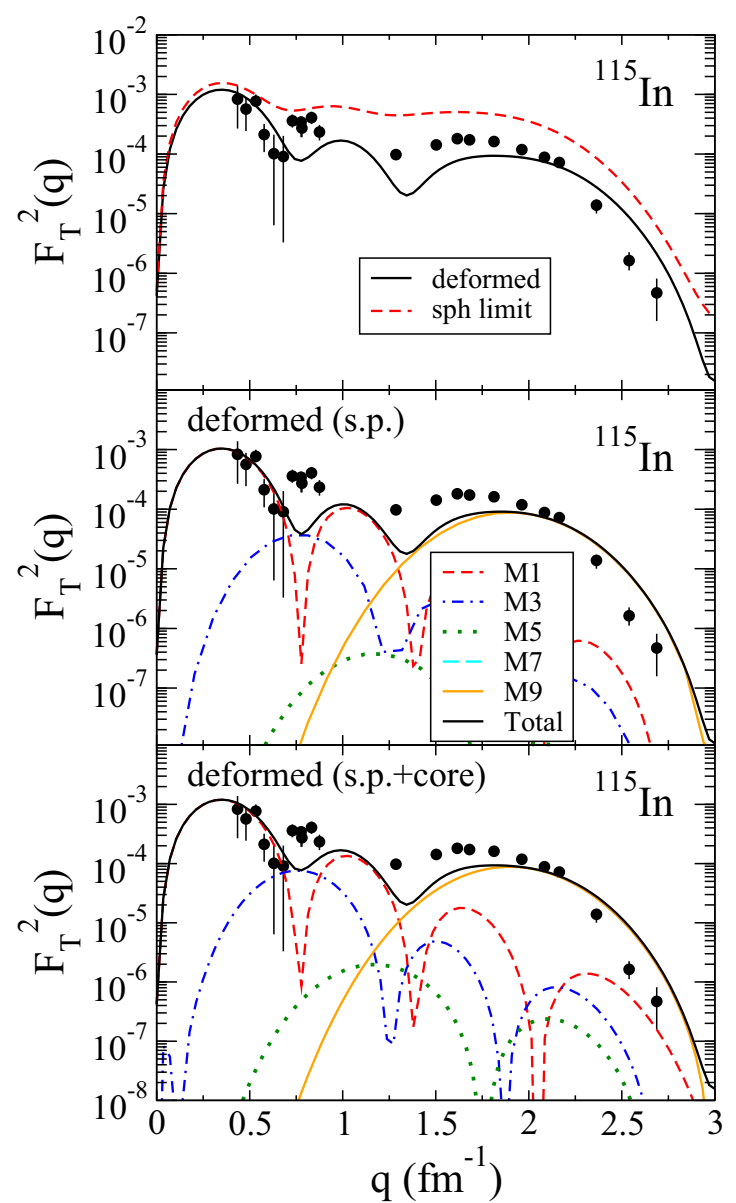

FIG. 9. Same as in Fig. 8, but for ${ }^{115}$ In $\left(I^{\pi}=9 / 2^{+}\right)$Data are taken from Ref. [7] and references therein.

moments from spherical and deformed calculations are close to each other with slightly larger values in the spherical case. On the other hand, in the cases of ${ }^{25} \mathrm{Mg}$ and ${ }^{29} \mathrm{Si}$, the behavior of M1 at low $q$ is very different, as well as the corresponding magnetic moments in Table I.

In summary, we have found that the geometrical factors in the deformed formalism reduce the multipoles with respect to the spherical ones and help to improve the agreement with experiment in all the deformed cases studied. These reduction factors appear naturally in the deformed formalism, whereas they have to be introduced ad hoc to fit the data in other approaches $[21,33]$. Collective effects manifest mainly at low$q$ values through M1 rotational multipoles. Although they are rather small in these calculations, they improve the agreement with experiment.

\section{CONCLUSIONS}

In this paper we have calculated magnetic form factors in elastic electron scattering from odd- $A$ nuclei within PWBA and within a deformed formalism, using wave functions from self-consistent $\mathrm{HF}+\mathrm{BCS}$ calculations with Skyrme forces.

We have recovered the spherical limit of these calculations and have compared the results with those obtained from spherical codes, finding a perfect agreement. We have 
shown that spherical nuclei are well described in this limit. NN correlations are included with natural orbits within the CDFM and are found to shift the tails of the form factors to higher momentum transfer, improving the agreement with experiment.

Then we proceed to calculate deformed nuclei and compare both spherical and deformed calculations with experiment. For these nuclei $\left({ }^{25} \mathrm{Mg},{ }^{29} \mathrm{Si},{ }^{59} \mathrm{Co}\right.$, and $\left.{ }^{115} \mathrm{In}\right)$, the deformed picture represents clearly an improvement of the agreement with the data, demonstrating the need for deformation degrees of freedom to describe these nuclei. The role of the collective rotation current of the core has been studied, showing that it manifests itself mainly in the M1 multipole, changing the profile of the form factor at low momentum transfer and improving in general the agreement with the measurements.

It is found that in odd- $A$ deformed nuclei, the main contribution to the magnetic form factor comes from the odd particle. This result is in contrast to the case of charge form factors, where all the nucleons contribute significantly. The collective effects in the deformed formulation enter in three different ways: (i) the deformation that modifies the singleparticle wave functions with respect to the wave functions in the spherical case; (ii) the collective current contributions through the rotational multipoles $\mathcal{F}_{R}^{M \lambda}$; and (iii) the reduction of the single-particle multipole contributions with respect to the spherical ones due to the strong particle-rotor coupling that manifests itself in the so-called geometrical factors. The effect of the rotational multipoles is more important in the low- $q$ region $\left(q<1 \mathrm{fm}^{-1}\right)$, where they interfere with the singleparticle contributions. This collective contribution from the deformed core must be included for a quantitative comparison with experiment.

We have shown that we can deal with spherical and deformed isotopes in a unified way using the same numerical methods and codes. It is also worth noting that, at variance with the approach followed in other works $[21,33]$, in this paper there is no fit of the coefficients weighting the various multipoles contributing to the total magnetic form factors. In the present formalism, the weights of the multipoles are directly given by the geometrical factors relating the intrinsic with the transition multipoles and therefore we do not introduce any adjustable parameter.

Once the capability of the model has been tested against data on magnetic form factors on spherical and deformed stable nuclei, we have a trustable formalism to explore the predictions on unstable nuclei.

\section{ACKNOWLEDGMENTS}

This work was supported by Ministerio de Ciencia, Innovación y Universidades (Spain) under Contracts No. FIS2014-51971-P and No. PGC2018-093636-B-I00. Three of the authors (M.K.G., A.N.A., and D.N.K.) are grateful for support of the Bulgarian Science Fund under Contract No. DFNI-T02/19.
[1] R. Hofstadter, Rev. Mod. Phys. 28, 214 (1956).

[2] T. de Forest and J. D. Walecka, Ad. Phys. 15, 1 (1966).

[3] T. W. Donnelly and J. D. Walecka, Ann. Rev. Nucl. Sci. 25, 329 (1975).

[4] H. De Vries, C. W. De Jager, and C. De Vries, At. Data Nucl. Data Tables 36, 495 (1987).

[5] S. Frullani and J. Mougey, Adv. Nucl. Phys. 14, 1 (1984).

[6] J. M. Udías, P. Sarriguren, E. Moya de Guerra, E. Garrido, and J. A. Caballero, Phys. Rev. C 48, 2731 (1993).

[7] T. W. Donnelly and I. Sick, Rev. Mod. Phys. 56, 461 (1984).

[8] E. Moya de Guerra, Phys. Rep. 138, 293 (1986).

[9] E. Moya de Guerra, Ann. Phys. (NY) 128, 286 (1980).

[10] P. von Neumann-Cosel, JPS Conf. Proc. 12, 010030 (2016).

[11] T. Suda and H. Simon, Prog. Part. Nucl. Phys. 96, 1 (2017).

[12] https://fair-center.eu/for-users/experiments/nustar/experiments/ elise.html

[13] http://www.riken.jp/en/research/labs/rnc/instrum_dev/scrit/

[14] A. N. Antonov et al., Nucl. Inst. Meth. A 637, 60 (2011).

[15] E. Enokizono, T. Onishi, and K. Tsukada, Nucl. Phys. News 28, 18 (2018).

[16] T. Ohnishi et al., Proc. Sci. INPC2016, 088 (2016).

[17] K. Tsukada, A. Enokizono, T. Ohnishi, K. Adachi, T. Fujita, M. Hara, M. Hori, T. Hori, S. Ichikawa, K. Kurita, K. Matsuda, T. Suda, T. Tamae, M. Togasaki, M. Wakasugi, M. Watanabe, and K. Yamada, Phys. Rev. Lett. 118, 262501 (2017).

[18] E. Garrido and E. Moya de Guerra, Nucl. Phys. A 650, 387 (1999); Phys. Lett. B 488, 68 (2000).
[19] A. N. Antonov, D. N. Kadrev, M. K. Gaidarov, E. Moya de Guerra, P. Sarriguren, J. M. Udias, V. K. Lukyanov, E. V. Zemlyanaya, and G. Z. Krumova, Phys. Rev. C 72, 044307 (2005).

[20] S. Karataglidis and K. Amos, Phys. Lett. B 650, 148 (2007).

[21] T. Dong, Z. Ren, and Y. Guo, Phys. Rev. C 76, 054602 (2007).

[22] X. Roca-Maza, M. Centelles, F. Salvat, and X. Viñas, Phys. Rev. C 78, 044332 (2008).

[23] X. Roca-Maza, M. Centelles, F. Salvat, and X. Viñas, Phys. Rev. C 87, 014304 (2013).

[24] J. Liu, C. Xu, and Z. Ren, Phys. Rev. C 95, 044318 (2017).

[25] J. Liu, C. Xu, S. Wang, and Z. Ren, Phys. Rev. C 96, 034314 (2017).

[26] R. A. Radhi, A. A. Alzubadi, and A. H. Ali, Phys. Rev. C 97, 064312 (2018).

[27] T. Liang, J. Liu, Z. Ren, C. Xu, and S. Wang, Phys. Rev. C 98, 044310 (2018).

[28] R. A. Radhi, N. T. Khalaf, and A. A. Najim, Nucl. Phys. A 724, 333 (2003).

[29] R. A. Radhi, Eur. Phys. J. A 34, 107 (2007).

[30] K. S. Jassim, A. A. Al-Sammarrae, F. I. Sharrad, and H. A. Kassim, Phys. Rev. C 89, 014304 (2014).

[31] A. A. Al-Sammarraie, F. I. Sharrad, N. Yusof, and H. A. Kassim, Phys. Rev. C 92, 034327 (2015).

[32] Z. Wang, Z. Ren, T. Dong, and C. Xu, Phys. Rev. C 90, 024307 (2014)

[33] Z. Wang, Z. Ren, T. Dong, and X. Guo, Phys. Rev. C 92, 014309 (2015). 
[34] J. Liu, X. Zhang, C. Xu, and Z. Ren, Nucl. Phys. A 948, 46 (2016).

[35] X. Guo, J. Liu, Z. Wang, and Z. Chi, Nucl. Phys. A 978, 1 (2018).

[36] E. Moya de Guerra and A. E. L. Dieperink, Phys. Rev. C 18, 1596 (1978).

[37] E. Moya de Guerra and S. Kowalski, Phys. Rev. C 22, 1308 (1980).

[38] E. Moya de Guerra and S. Kowalski, Phys. Rev. C 20, 357 (1979).

[39] E. Graca, P. Sarriguren, D. Berdichevsky, D. W. L. Sprung, E. Moya de Guerra, and M. Nishimura, Nucl. Phys. A 483, 77 (1988).

[40] P. Sarriguren, E. Graca, D. W. L. Sprung, E. Moya de Guerra, and D. Berdichevsky, Phys. Rev. C 40, 1414 (1989).

[41] K. Heyde, P. von Neumann-Cosel, and A. Richter, Rev. Mod. Phys. 82, 2365 (2010).

[42] P. Sarriguren, E. Moya de Guerra, R. Nojarov, and A. Faessler, J. Phys. G 20, 315 (1994).

[43] F. N. Rad, W. Bertozzi, S. Kowalski, C. P. Sargent, C. F. Williamson, M. V. Hynes, B. Norum, B. Peterson, T. Sasanuma, and W. Turchinetz, Phys. Rev. Lett. 45, 1758 (1980).

[44] D. Berdichevsky, P. Sarriguren, E. Moya de Guerra, M. Nishimura, and D. W. L. Sprung, Phys. Rev. C 38, 338 (1988).

[45] E. Moya de Guerra, P. Sarriguren, J. A. Caballero, M. Casas, and D. W. L. Sprung, Nucl. Phys. A 529, 68 (1991).

[46] G. G. Simon, C. Schmitt, F. Borkowski, and V. H. Walther, Nucl. Phys. A 333, 381 (1980).

[47] H. Chandra and G. Sauer, Phys. Rev. C 13, 245 (1976).

[48] D. R. Yennie, D. G. Ravenhall, and R. N. Wilson, Phys. Rev. 95, 500 (1954).

[49] P. Sarriguren and E. Moya de Guerra, Phys. Rev. C 30, 2105 (1984); M. Nishimura, E. Moya de Guerra, and D. W. L. Sprung, Nucl. Phys. A 435, 523 (1985); M. Nishimura, D. W. L. Sprung, and E. Moya de Guerra, Phys. Lett. B 161, 235 (1985).

[50] D. Vautherin and D. M. Brink, Phys. Rev. C 5, 626 (1972); D. Vautherin, ibid. 7, 296 (1973).

[51] N. Schunck, J. Dobaczewski, J. McDonnell, J. Moré, W. Nazarewicz, J. Sarich, and M. V. Stoitsov, Phys. Rev. C 81, 024316 (2010).

[52] S. Perez-Martin and L. M. Robledo, Phys. Rev. C 78, 014304 (2008).

[53] M. Bender, J. Dobaczewski, J. Engel, and W. Nazarewicz, Phys. Rev. C 65, 054322 (2002).
[54] M. Zalewski, J. Dobaczewski, W. Satula, and T. R. Werner, Phys. Rev. C 77, 024316 (2008).

[55] E. Chabanat, P. Bonche, P. Haensel, J. Meyer, and R. Schaeffer, Nucl. Phys. A 635, 231 (1998).

[56] A. N. Antonov, P. E. Hodgson, and I. Zh. Petkov, Nucleon Momentum and Density Distributions in Nuclei (Clarendon Press, Oxford, 1988); Nucleon Correlations in Nuclei (SpringerVerlag, Berlin, 1993).

[57] D. N. Kadrev, A. N. Antonov, M. V. Stoitsov, and S. S. Dimitrova, Int. J. Mod. Phys. E 5, 717 (1996).

[58] P.-O. Löwdin, Phys. Rev. 97, 1474 (1955).

[59] A. N. Antonov, V. A. Nikolaev, and I. Zh. Petkov, Bulg. J. Phys. 6, 151 (1979); Z. Phys. A 304, 239 (1982).

[60] A. N. Antonov, E. N. Nikolov, I. Zh. Petkov, C. V. Christov, and P. E. Hodgson, Nuovo Cimento A 102, 1701 (1989).

[61] A. N. Antonov, D. N. Kadrev, and P. E. Hodgson, Phys. Rev. C 50, 164 (1994).

[62] J. J. Griffin and J. A. Wheeler, Phys. Rev. 108, 311 (1957).

[63] I. Angeli and K. P. Marinova, At. Data Nucl. Data Tables 99, 69 (2013).

[64] N. J. Stone, At. Data Nucl. Data Tables 90, 75 (2005).

[65] H. P. Blok and J. H. Heisenberg, in Computational Nuclear Physics 1: Nuclear Structure, edited by K. Langanke, J. A. Maruhn, and S. E. Koonin (Springer-Verlag, Berlin, 1991).

[66] M. V. Hynes, H. Miska, B. Norum, W. Bertozzi, S. Kowalski, F. N. Rad, C. P. Sargent, T. Sasanuma, W. Turchinetz, and B. L. Berman, Phys. Rev. Lett. 42, 1444 (1979).

[67] H. Baghaei, A. Cichocki, J. B. Flanz, M. Frodyma, B. Frois, Y. Han, R. S. Hicks, J. Martino, R. A. Miskimen, C. N. Papanicolas, G. A. Peterson, S. Platchkov, S. Raman, S. H. Rokni, and T. Suzuki, Phys. Rev. C 42, 2358 (1990).

[68] K. Arita, A. Enomoto, S. Oguro, Y. Mizuno, T. Nakazato, S. Ohsawa, T. Saito, T. Terasawa, and Y. Torizuka, Phys. Rev. C 23, 1482 (1981).

[69] S. K. Platchkov et al., Phys. Lett. B 131, 301 (1983).

[70] R. C. York and G. A. Peterson, Phys. Rev. C 19, 574 (1979).

[71] S. K. Platchkov, J. B. Bellicard, J. M. Cavedon, B. Frois, D. Goutte, M. Huet, P. Leconte, P. Xuan-Ho, P. K. A. de Witt Huberts, L. Lapikás, and I. Sick, Phys. Rev. C 25, 2318 (1982).

[72] H. Euteneuer, H. Rothhaas, O. Schwentker, J. R. Moreira, C. W. de Jager, L. Lapikás, H. de Vries, J. Flanz, K. Itoh, G. A. Peterson, D. V. Webb, W. C. Barber, and S. Kowalski, Phys. Rev. C 16, 1703 (1977).

[73] H. de Vries, G. J. C. Van Niftrik, and L. Lapikás, Phys. Lett. B 33, 403 (1970). 